\title{
Phase II study of oxaliplatin, UFT, and leucovorin in patients with metastatic gastric cancer
}

\author{
Ester J.M. Siemerink ${ }^{1}$, Annemieke F.J. Drenth ${ }^{2}$, Nanno H. Mulder ${ }^{1}$, John T.M. Plukker ${ }^{2}$, \\ and GeKe A.P. Hospers ${ }^{1}$ \\ ${ }^{1}$ Department of Medical Oncology, University Medical Center Groningen, University of Groningen, P.O. Box 30.001, 9700 RB Groningen, \\ the Netherlands \\ ${ }^{2}$ Department of Surgery, University of Groningen and University Medical Center Groningen, Groningen, the Netherlands
}

\begin{abstract}
Background. The present study evaluated the efficacy and safety of oxaliplatin, UFT, and leucovorin in metastatic gastric cancer.

Methods. Patients received intravenous oxaliplatin $130 \mathrm{mg} / \mathrm{m}^{2}$ on day 1 , followed by oral UFT capsules $\left(350 \mathrm{mg} / \mathrm{m}^{2}\right.$ per day) and leucovorin tablets ( $90 \mathrm{mg} /$ day), every $8 \mathrm{~h}$, for 14 days, in a 3-week cycle.

Results. Twenty-three patients (61\% with $\geq 2$ metastatic sites), median age of 60 years (range, 39-69 years) were entered. Based on intention-to-treat analysis, one complete response and seven partial responses were found, resulting in an overall response rate $(\mathrm{RR})$ of $35 \%(95 \%$ confidence interval [CI], 16-54), a median time to progression of 4 months (95\% CI, 0.5-7.5), and a median overall survival (OS) of 8 months $(95 \%$ CI, 4.5-11.5). The 1-year survival rate was $26 \%$. Three patients did not complete the first course of 2 weeks; 1 died suddenly on day 16 with fatal lung embolism; 1 had rapid progressive disease and 1 experienced gastric hemorrhage on day 15 both these patients withdrew. In the 20 patients assessable for toxicity no grade 4 toxicity occurred, grade 3 toxicity consisted of anemia in 1, diarrhea in 2 , and neurotoxicity in 3 patients. No hand-foot syndrome (HFS) occurred.

Conclusion. Oxaliplatin is an effective drug in gastric cancer, but, as previously reported, its feasibility in combination with capecitabine is hampered due to combined hand-foot-based toxicity. The present phase II study of a combination of oxaliplatin with UFT and leucovorin appears to have efficacy and tolerability comparable to two other drug regimens used in gastric cancer, without the HFS problem.
\end{abstract}

Key words Metastatic gastric cancer - Chemotherapy $\cdot$ Handfoot syndrome $\cdot$ Outpatient setting

Offprint requests to: G.A.P. Hospers

Received: October 7, 2009 / Accepted: January 28, 2010

\section{Introduction}

Gastric cancer is the world's second leading cause of cancer-related death. Unfortunately, most patients present with an advanced stage of disease, with a dismal outcome. Even after apparently curative resections, local recurrences or distant metastasis occur in up to $60 \%$ of the patients $[1,2]$.

In this palliative situation patients do benefit from combination chemotherapy compared to best supportive care, as it shows a modest survival benefit, with improvement of quality of life [3-6]. 5-Fluorouracil (5-FU) is still one of the main chemotherapeutic agents used in advanced gastric cancer, with a response rate (RR) as a single agent of $21 \%$ and a median survival of 6-7 months; while at the same time epirubicin, cisplatin, and 5-FU (ECF) is the most widely used combination regimen. With an overall RR of ECF of $40 \%-45 \%$ and a median survival of around $8-10$ months it is considered a reference regimen, especially in Europe [2, 7-10]. In the context of quality of life, the observed toxicity, prolonged hospital time (due to cisplatin infusion), and the risks of central venous access devices and ambulatory infusion pumps make the use of the combination regimens instead of monochemotherapy debatable. There is a need for an effective chemotherapy combination which is less toxic and can be easily administered in an outpatient setting. In order to improve convenience and tolerability three oral fluoropyrimidines have been developed [11, 12]. One of them is UFT, a combination of tegafur, an oral prodrug of 5-FU which is slowly metabolized in the liver into the active drug 5-FU, and uracil, a competitive antagonist for dihydropyrimidine dehydrogenase (DPD), in a 1:4 $\mathrm{M}$ ratio. Uracil inhibits the degradation of 5-FU because it competes with 5-FU for DPD, leading to higher intratumoral 5-FU levels. This combination drug has an RR of about $16 \%-57 \%$ and a median overall survival (OS) of 5.8 to 15 months and 
is less toxic as far as myelosupression, stomatitis, diarrhea, and hand-foot syndrome (HFS) are concerned [11-16]. Clinical studies in metastatic colon cancer have shown that UFT has an efficacy profile comparable to that of intravenous bolus administration of 5-FU [17, 18]. Capecitabine, the oral fluoropyrimidine most often used worldwide, has a toxicity and efficacy profile similar to that of UFT, except that HFS, which is rarely observed with UFT, occurs in more than half of all patients treated with capecitabine $[19,20]$. A third oral fluoropyrimidine, S-1, has not been registered in Europe, and is licensed in Korea and Japan [11].

Oxaliplatin is a third-generation platinum derivative that inhibits replication and transcription by the formation of DNA adducts. It has shown antitumor activity as monotherapy or in combination with 5-FU and leucovorin (LV) in various solid tumors, including gastric cancer. In comparison with cisplatin it has a more favorable toxicity profile, with substantially lower rates of myelosuppression, nephrotoxicity, and ototoxicity, but with at least equivalent activity [21-24]. The duration of intravenous hydration required with cisplatin use is not required for oxaliplatin, thereby facilitating outpatient administration. In combination with other chemotherapeutic agents, the UFT/LV schedules that are often used are UFT doses of $300-400 \mathrm{mg} / \mathrm{m}^{2}$ per day, and LV doses of $25-500 \mathrm{mg}$, for 1-14 treatment days per 21 days or 1-28 treatment days per 35 days [11]. In combination with a fluoropyrimidine, oxaliplatin is given in a 2- or 3-weekly schedule at an intravenous (i.v.) dose of, respectively, 85 or $130 \mathrm{mg} / \mathrm{m}^{2}$ [25-27].

Based on these promising data of two different active agents with little overlap in terms of key side effects, we conducted a phase II study of oxaliplatin $\left(130 \mathrm{mg} / \mathrm{m}^{2}\right.$ on day 1), UFT capsules (350 $\mathrm{mg} / \mathrm{m}^{2}$ per day), LV tablets (90 $\mathrm{mg}$ /day), every $8 \mathrm{~h}$, for 14 days, in a 3 -week cycle in patients with metastatic gastric cancer, in an outpatient setting, to investigate the antitumor activity and toxicity of this combination regimen.

\section{Patients and methods}

\section{Eligibility}

Between February 2004 and September 2008, patients with histologically confirmed metastatic gastric cancer with at least one measurable lesion according to the response evaluation criteria in solid tumors (RECIST) were considered to be eligible in this open, noncomparative phase II study.

All patients were aged more than 18 years, with an ambulatory performance status of $0-1$ on the Eastern Cooperative Oncology Group (ECOG) scale and a life expectancy of more than 3 months. Laboratory accep- tance parameters included adequate hematological (white blood cell count $4.0 \times 10^{9} / 1$ ), hepatic (serum bilirubin $<1.5 \times$ upper limit of normal), and renal (calculated creatinine clearance of $>50 \mathrm{ml} / \mathrm{min}$ ) function. Exclusion criteria consisted of: bone metastasis or effusions as the only manifestation of disease; clinical signs of brain metastasis; concurrent radiation therapy or previous chemotherapy; previous or current malignancies at other sites; evidence of serious active infections; severe cardiac and/or pulmonary failure; and pregnant or lactating women.

The study was approved by the ethics review board of our hospital (METc 2004.016) and was carried out in accordance with the Declaration of Helsinki principles. Written informed consent was obtained from all patients at a 7-day interval after thorough information had been given about the study.

\section{Pretreatment evaluation}

Prestudy assessment consisted of full medical history, vital signs, and physical examination. Further investigations included hematological and blood chemistry testing, electrocardiogram, chest X-ray, and a computed tomography (CT) scan of the abdomen.

\section{Treatment schedule}

Oxaliplatin $\left(130 \mathrm{mg} / \mathrm{m}^{2}\right)$ was administered by a 2 -h i.v. infusion on day 1 followed by oral UFT capsules (350 $\mathrm{mg} / \mathrm{m}^{2}$ per day) and $\mathrm{LV}$ tablets $(90 \mathrm{mg} /$ day) taken simultaneously every $8 \mathrm{~h}$ from the evening of day 1 to the morning of day 15 , followed by a 7-day treatmentfree interval in a 3-week cycle. After two and four cycles, patients with a tumor response or stable disease continued on chemotherapy, receiving a maximum of six cycles.

\section{Dose modification}

In patients with nonhematological toxicity of grade 2 or greater, treatment was postponed until toxicity resolved to grade 1 or less; the next UFT dose was reduced by $25 \%$ in patients with grade $3-4$ toxicity. With hematological toxicity of grade 2 or greater, treatment was withheld until the white blood cell count was $3.0 \times 10^{9} / 1$ or greater and the platelet count was 100 $\times 10^{9} / 1$ or greater. The oxaliplatin dose was reduced by $25 \%$ in patients with persistent ( $\geq 14$ days) paresthesia or temporary painful paresthesia or functional impairment. Treatment was discontinued in patients with grade 4 nonhematological toxicity or nonrecovery of persistent paresthesia, and in those with nonhematological toxicity of grade 1 or greater despite a 2-week delay. 


\section{Treatment response and toxicity}

Tumor response was evaluated on a CT scan after cycles 2 and 4 , and after the treatment was finished. The tumor responses were classified according to the RECIST. All patients were examined on days 1 and 15 of each cycle for vital signs, including physical examination, ECOG performance status, and complete blood counts and biochemical tests. Toxicity was evaluated according to the National Cancer Institute Common Toxicity Criteria (NCI CTC), version 2.0.

\section{Statistical analyses}

For the determination of the number of patients required in a preliminary and follow-up trial of a new chemotherapeutic agent, Gehan's two-stage design was used [28]. We expected a response rate of $30 \%$ or greater. If no objective response was seen in the first 12 patients, then the probability of a response rate of $30 \%$ or greater would be below $5 \%$ and no additional patients would be entered. If, in the first 12 patients more than four responses occurred, 8 additional patients would therefore be required for the evaluability of response. With a dropout frequency of $10 \%, 23$ patients had to be included to estimate the $95 \%$ confidence interval (CI) for a true response rate with a maximum width of $38 \%$.

\section{Results}

\section{Patient characteristics}

During the study period a total of 23 patients, 21 men, and two women, with a median age of 60 years (range, 39-69 years) were enrolled. Eighty percent of these patients had a performance score of up to 1 at the start of treatment. Most patients (21/23) had a metastatic disease at the time of diagnosis, while 2 patients developed metastatic disease after surgical resection of the primary tumor. The most common metastatic sites were liver, lung, bone, or peritoneum (83\% of the patients), while $17 \%$ of the patients had only lymph node metastasis. Two or more metastatic sites occurred in $61 \%$ of the patients. At the evaluation in November 2009 all 23 patients had died.

\section{Efficacy and survival}

Twenty of the 23 patients were assessable for toxicity, while the remaining 3 did not complete the first course of 3 weeks. One patient died suddenly on day 16 with a clinical diagnosis of fatal lung embolism, and a second patient had rapid progression of pulmonary metastasis and withdrew. The third patient, who still had his primary tumor in situ, experienced a gastric hemorrhage on day
15 and withdrew. All efficacy data are reported using the intention-to-treat patient population.

The overall RR was 35\% (95\% CI, 16-54); one patient had a complete remission, seven patients had a partial response, eight patients had stable disease, and four patients had progressive disease. The median time to progression was 4 months $(95 \% \mathrm{CI}, 0.5-7.5)$ and the median OS was 8 months (95\% CI, 4.5-11.5); see Figs. 1 and 2 . The 1 -year survival rate was $26 \%$. Three initially responding patients in this study received second-line chemotherapy. One patient progressed after 1.5 years and was treated with irinotecan because of disease progression; however, this treatment was without an objective response. The two other patients progressed, after 6 and 8 months, respectively, and were treated with the oral fluoropyrimidine capecitabine; however, this treatment was also without an objective response.

\section{Toxicity}

The patients received a total number of 86 cycles (median, 3.5 cycles; range, 1-6 cycles). Nine patients received the maximum of 6 cycles. The most common

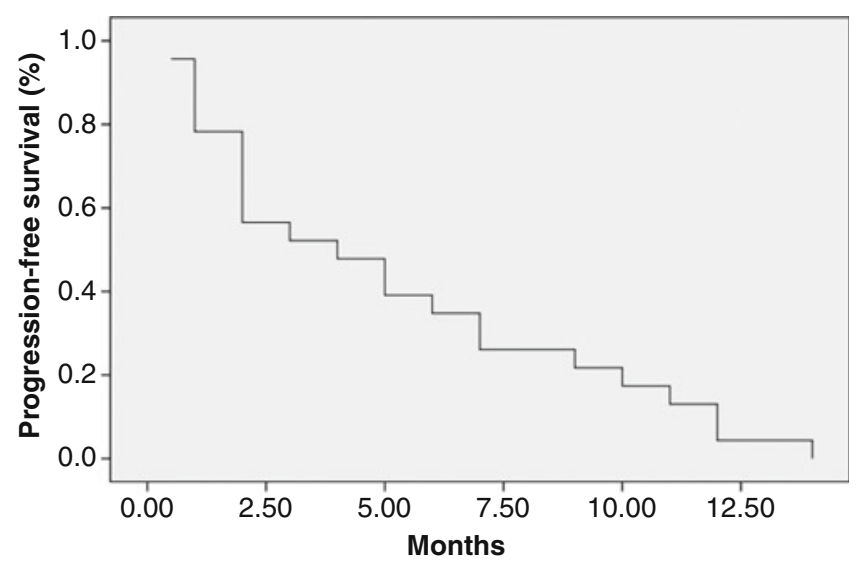

Fig. 1. Progression-free survival (months)

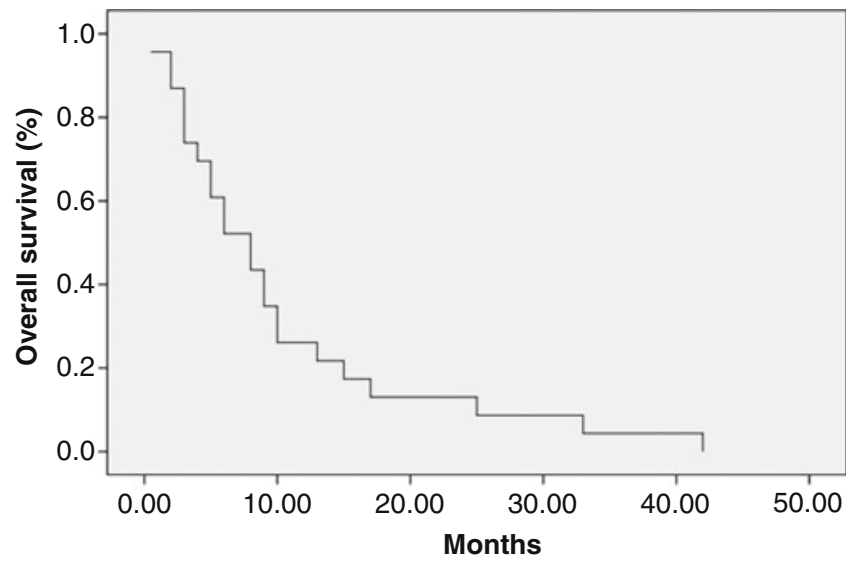

Fig. 2. Overall survival (months) 
Table 1. Toxicity

\begin{tabular}{lcccc}
\hline NCI-CTC grade & 1 & 2 & 3 & 4 \\
\hline Hand-foot syndrome & - & - & - & - \\
Neuropathy & 2 & 6 & 3 & - \\
Diarrhea & 1 & 3 & 2 & - \\
Nausea & 4 & 3 & - & - \\
Weight loss & 5 & - & - & - \\
Thrombocytopenia & - & 8 & - & - \\
Leukopenia & 1 & - & - & - \\
Anemia & 1 & 1 & 1 & - \\
ASAT & 2 & 1 & - & - \\
ALAT & 2 & 3 & - & - \\
Bilirubin & - & - & - & - \\
\hline
\end{tabular}

NCI-CTC, National Cancer Institute Common Toxicity Criteria; ASAT, aspartate aminotransferase; ALAT, alanine aminotransferase

reported toxicity was oxaliplatin-related sensory neuropathy, in 11 patients. Usually this was mild and reversible, with a need for dose reduction of oxaliplatin in only 3 patients. Other nonhematological toxicities consisted of diarrhea in 6 patients ( 2 with grade 3 toxicity), grade $1 / 2$ nausea in 7 patients, and grade 1 weight loss in 5 patients. Hematological toxicities observed were grade 2 anemia in 1 patient and grade 2 thrombocytopenia in 8 patients, and grade 3 anemia in 1 patient. No grade 4 hematological or nonhematological toxicity was reported (Table 1). Eventually, treatment-related reasons for discontinuation were: persistent thrombocytopenia after 2 weeks of postponing the next cycle in 3 patients (after cycles 3,4 , and 5, respectively), grade 3 diarrhea in 2 patients, with the need for hospitalization and clinical worsening in 1 patient and recurrent diarrhea after dose reduction in the previous cycle in the other patient. The gastric hemorrhage that occurred on day 15 may have been treatment-related. The fatal lung embolism that occurred in cycle one did not seem to be treatment-related.

Treatment was also discontinued due to clinical tumor progression in four patients, and due to tumor progression on CT scan in three patients. After discontinuation because of prolonged thrombocytopenia, two patients continued off study on mono UFT/LV.

\section{Discussion}

There is currently no universal standard regimen for the treatment of advanced gastric cancer. With two drugs commonly used drug regimens often consist of a cisplatin and fluorouracil combination. In a palliative situation this regimen is not very patient-friendly, as hospitalization is often required, as is the use of continuous intravenous infusion.

Compared with the overall RR in the literature, with ECF and epirubicin, oxaliplatin, capecitabin (EOX) regimens showing RRs of $38 \%-47 \%$ and a median survival of around 8-11 months, the results of our study, showing an overall RR of $35 \%$ and median OS of 8.0 months, are similar but on the lower boundary $[2,7-10]$. This may be due to the relatively large number of patients showing early progression in our study who could not complete the first course. Our study also included patients with a relatively high stage of advanced disease, as $61 \%$ of the patients had two or more metastatic sites, compared with $36 \%-40 \%$ of the patients in the randomized ECF for advanced and locally advanced esophagogastric cancer 2 (REAL-2) study [7]. Based on the remaining 20 evaluable patients in the present study, our combination chemotherapy of oxaliplatin, UFT, and LV showed acceptable antitumor activity, with an RR of $40 \%$ and a median OS of 9 months, and could be safely administered on an outpatient basis.

Several phase II-III studies have already indicated that oxaliplatin-based doublets may represent an effective and well-tolerated treatment option, compared to cisplatin, for patients with advanced gastric cancer [7, 29-32]. In a study by Cunningham [7], oxaliplatin, compared to cisplatin, showed significantly less grade $3 / 4$ neutropenia, alopecia, and thromboembolism, with significantly more grade $3 / 4$ diarrhea and peripheral neuropathy. This prompted these authors to the conclusion that oxaliplatin could replace cisplatin. The results of a study by Al-Batran et al. [33] are consistent with the REAL-2 data that oxaliplatin is at least as effective as cisplatin in patients with advanced gastric cancer. The results of these two studies [7,33] are comparable regarding neurotoxicity, and are comparable with the generally mild and reversible neurotoxicity observed in our study, in which all patients with grade 3 neurotoxicity could continue on treatment after dose reduction. As patients prefer oral to intravenous therapy provided that no more side effects occur and efficacy is not compromised, UFT and LV are a logical alternative to intravenous 5-FU [15]. Different phase II studies have investigated the efficacy of UFT with LV in combination with another agent [11,34-36]. These efficacy results are comparable with the RR of $35 \%$ and confidence interval of $16 \%-54 \%$ in our study.

More often studies in patients with advanced gastric cancer use the oral fluoropyrimidine capecitabine. The REAL-2 data and the study of Okines et al. showed at least similar efficacy of intravenous fluorouracil and the oral fluoropyrimidine capecitabine [7,37]. The all-grade HFS was high in all groups [ECF, 29.8\%; epirubicin, cisplatin, capecitabin (ECX), 45.9\%; epirubicin, oxaliplatin, 5-FU (EOF), 28.9\%; EOX, 39.3\%]. Moreover, in the patients receiving capecitabine with epirubicin and cisplatin, significantly more grade $3 / 4$ HFS was observed (grade $3 / 4 ; 10.3 \%$ ) than in the ECF group (grade $3 / 4$; $4.3 \%$ ) [7]. Another recently published phase II study 
also emphasized the problem of HFS, which occurred in $39 \%$ of the patient population receiving capecitabine and oxaliplatin [32]. The problem of hand-foot-based toxicity interfering with quality of life in a palliative situation is disturbing. In our study, as in many other studies with UFT-based combinations, patients reported hardly any HFS $(<0.01 \%)$. This observation forms the basis for replacing the oral fluoropyrimidine capecitabine with UFT. The efficacies of two-drug combinations with UFT are comparable with those of other capecitabinebased regimens, with reported overall RRs of 35\%-49\% and median OS rates of 6-11 months [2, 11, 30, 38].

For the future, new promising targeted biological agents, often combined with chemotherapeutic drugs, are being investigated. Although biological agents are seemingly less toxic, epidermal growth factor receptor (EGFR) inhibitors can cause severe paronychia, and more commonly, acneiform rash [39]. Based on the toxicity profiles of UFT and capecitabine, it seems to be more logical to combine UFT with these new targeted biologicals.

In conclusion, based on the REAL-2 data, oxaliplatin has an important place in the first-line treatment of advanced gastric cancer [7]. However, the use of oxaliplatin in combination with capecitabine has led to an increasing number of patients with hand-foot problems. Therefore, combining a UFT/LV-based regimen with oxaliplatin in patients with advanced gastric cancer has the advantage of a good tolerability profile, with no HFS, and the regimen can be easily administered in an outpatient setting. For future studies with the new targeted agents, a combination of such agents with UFT would seem to be superior in dealing with the problem of hand-foot toxicity.

\section{References}

1. Wilke H, Preusser P, Fink U, Achterrath W, Mayer HJ, Stahl M, et al. New developments in the treatment of gastric carcinoma. Cancer Treat Res 1991;55:363-73.

2. Rivera F, Vega-Villegas ME, Lopez-Brea MF. Chemotherapy of advanced gastric cancer. Cancer Treat Rev 2007;33:315-24.

3. Glimelius B, Ekstrom K, Hoffman K, Graf W, Sjoden PO, Haglund $\mathrm{U}$, et al. Randomized comparison between chemotherapy plus best supportive care with best supportive care in advanced gastric cancer. Ann Oncol 1997;8:163-8.

4. Murad AM, Santiago FF, Petroianu A, Rocha PR, Rodrigues MA, Rausch M. Modified therapy with 5-fluorouracil, doxorubicin, and methotrexate in advanced gastric cancer. Cancer 1993;72:3741.

5. Pyrhonen S, Kuitunen T, Nyandoto P, Kouri M. Randomised comparison of fluorouracil, epidoxorubicin and methotrexate (FEMTX) plus supportive care with supportive care alone in patients with non-resectable gastric cancer. Br J Cancer 1995;71: 587-91.

6. Wohrer SS, Raderer M, Hejna M. Palliative chemotherapy for advanced gastric cancer. Ann Oncol 2004;15:1585-95.
7. Cunningham D, Starling N, Rao S, Iveson T, Nicolson M, Coxon $\mathrm{F}$, et al. Capecitabine and oxaliplatin for advanced esophagogastric cancer. N Engl J Med 2008;358:36-46.

8. Ross P, Nicolson M, Cunningham D, Valle J, Seymour M, Harper $\mathrm{P}$, et al. Prospective randomized trial comparing mitomycin, cisplatin, and protracted venous-infusion fluorouracil (PVI 5-FU) with epirubicin, cisplatin, and PVI 5-FU in advanced esophagogastric cancer. J Clin Oncol 2002;20:1996-2004.

9. Webb A, Cunningham D, Scarffe JH, Harper P, Norman A, Joffe JK, et al. Randomized trial comparing epirubicin, cisplatin, and fluorouracil versus fluorouracil, doxorubicin, and methotrexate in advanced esophagogastric cancer. J Clin Oncol 1997;15: 261-7.

10. Wagner AD, Grothe W, Haerting J, Kleber G, Grothey A, Fleig WE. Chemotherapy in advanced gastric cancer: a systematic review and meta-analysis based on aggregate data. J Clin Oncol 2006;24:2903-9.

11. Aykan NF, Idelevich E. The role of UFT in advanced gastric cancer. Ann Oncol 2008;19:1045-52.

12. Pazdur R, Hoff PM, Medgyesy D, Royce M, Brito R. The oral fluorouracil prodrugs. Oncology (Williston Park) 1998;12:4851.

13. Ravaud A, Borner M, Schellens JH, Geoffrois L, Schoffski BP, Kroon K, et al. UFT and leucovorin in first-line chemotherapy for patients with metastatic gastric cancer. An Early Clinical Studies Group (ECSG)/European Organization for Research Treatment of Cancer (EORTC) phase II trial. Eur J Cancer 2001;37: $1642-7$.

14. Takiuchi H, Ajani JA. Uracil-tegafur in gastric carcinoma: a comprehensive review. J Clin Oncol 1998;16:2877-85.

15. Kopec JA, Yothers G, Ganz PA, Land SR, Cecchini RS, Wieand HS, et al. Quality of life in operable colon cancer patients receiving oral compared with intravenous chemotherapy: results from National Surgical Adjuvant Breast and Bowel Project Trial C-06. J Clin Oncol 2007;25:424-30.

16. Kim YH, Cheong SK, Lee JD, Park JS, Shin SW, Kim JS. Phase II trial of oral UFT and leucovorin in advanced gastric carcinoma. Am J Clin Oncol 1996;19:212-6.

17. Carmichael J, Popiela T, Radstone D, Falk S, Borner M, Oza A, et al. Randomized comparative study of tegafur/uracil and oral leucovorin versus parenteral fluorouracil and leucovorin in patients with previously untreated metastatic colorectal cancer. J Clin Oncol 2002;20:3617-27.

18. Douillard JY, Hoff PM, Skillings JR, Eisenberg P, Davidson N, Harper P, et al. Multicenter phase III study of uracil/tegafur and oral leucovorin versus fluorouracil and leucovorin in patients with previously untreated metastatic colorectal cancer. J Clin Oncol 2002;20:3605-16.

19. Twelves C, Wong A, Nowacki MP, Abt M, Burris H 3rd, Carrato A, et al. Capecitabine as adjuvant treatment for stage III colon cancer. N Engl J Med 2005;352:2696-704.

20. Lembersky BC, Wieand HS, Petrelli NJ, O'Connell MJ, Colangelo LH, Smith RE, et al. Oral uracil and tegafur plus leucovorin compared with intravenous fluorouracil and leucovorin in stage II and III carcinoma of the colon: results from National Surgical Adjuvant Breast and Bowel Project Protocol C-06. J Clin Oncol 2006;24:2059-64.

21. Al-Batran SE, Atmaca A, Hegewisch-Becker S, Jaeger D, Hahnfeld S, Rummel MJ, et al. Phase II trial of biweekly infusional fluorouracil, folinic acid, and oxaliplatin in patients with advanced gastric cancer. J Clin Oncol 2004;22:658-63.

22. Cassidy J, Tabernero J, Twelves C, Brunet R, Butts C, Conroy T, et al. XELOX (capecitabine plus oxaliplatin): active first-line therapy for patients with metastatic colorectal cancer. J Clin Oncol 2004;22:2084-91.

23. Park YH, Kim BS, Ryoo BY, Yang SH. A phase II study of capecitabine plus 3-weekly oxaliplatin as first-line therapy for patients with advanced gastric cancer. Br J Cancer 2006;94: 959-63. 
24. Woynarowski JM, Faivre S, Herzig MC, Arnett B, Chapman WG, Trevino AV, et al. Oxaliplatin-induced damage of cellular DNA. Mol Pharmacol 2000;58:920-7.

25. André T, Boni C, Mounedji-Boudiaf L, Navarro M, Tabernero J, Hickish T, et al. Multicenter International Study of Oxaliplatin/5Fluorouracil/Leucovorin in the Adjuvant Treatment of Colon Cancer (MOSAIC) Investigators. Oxaliplatin, fluorouracil, and leucovorin as adjuvant treatment for colon cancer. N Engl J Med 2004;350:2343-51.

26. Borner MM, Dietrich D, Stupp R, Morant R, Honegger H, Wernli $\mathrm{M}$, et al. Phase II study of capecitabine and oxaliplatin in first- and second-line treatment of advanced or metastatic colorectal cancer. J Clin Oncol. 2002;20:1759-66.

27. Maindrault-Goebel F, de Gramont A, Louvet C, André T, Carola E, Mabro M, et al. High-dose intensity oxaliplatin added to the simplified bimonthly leucovorin and 5-fluorouracil regimen as second-line therapy for metastatic colorectal cancer (FOLFOX 7). Eur J Cancer 2001;37:1000-5.

28. Gehan EA. The determination of the number of patients required in a preliminary and a follow-up trial of a new chemotherapeutic agent. J Chronic Dis 1961;13:346-53.

29. De VF, Orditura M, Matano E, Bianco R, Carlomagno C, Infusino $\mathrm{S}$, et al. A phase II study of biweekly oxaliplatin plus infusional 5-fluorouracil and folinic acid (FOLFOX-4) as first-line treatment of advanced gastric cancer patients. Br J Cancer 2005;92:1644-9.

30. Jatoi A, Murphy BR, Foster NR, Nikcevich DA, Alberts SR, Knost $\mathrm{JA}$, et al. Oxaliplatin and capecitabine in patients with metastatic adenocarcinoma of the esophagus, gastroesophageal junction and gastric cardia: a phase II study from the North Central Cancer Treatment Group. Ann Oncol 2006;17:29-34.

31. Lordick F, Lorenzen S, Stollfuss J, Vehling-Kaiser U, Kullmann F, Hentrich M, et al. Phase II study of weekly oxaliplatin plus infusional fluorouracil and folinic acid (FUFOX regimen) as first-line treatment in metastatic gastric cancer. Br J Cancer 2005;93: $190-4$.
32. Park YH, Lee JL, Ryoo BY, Ryu MH, Yang SH, Kim BS, et al. Capecitabine in combination with oxaliplatin (XELOX) as a firstline therapy for advanced gastric cancer. Cancer Chemother Pharmacol 2008;61:623-9.

33. Al-Batran SE, Hartmann JT, Probst S, Schmalenberg H, Hollerbach S, Hofheinz R, et al. Phase III trial in metastatic gastroesophageal adenocarcinoma with fluorouracil, leucovorin plus either oxaliplatin or cisplatin: a study of the Arbeitsgemeinschaft Internistische Onkologie. J Clin Oncol 2008;26:1435-42.

34. Feliu J, Gonzalez BM, Garcia-Giron C, Espinosa E, GarciaAlfonso P, Belon J, et al. Treatment of patients with advanced gastric carcinoma with the combination of etoposide plus oral tegafur modulated by uracil and leucovorin. A phase II study of the ONCOPAZ Cooperative Group. Cancer 1996;78:211-6.

35. Suga S, Iwase H, Shimada M, Nishio Y, Ichihara T, Ichihara S, et al. Neoadjuvant chemotherapy in scirrhous cancer of the stomach using uracil and tegafur and cisplatin. Intern Med 1996;5:930-6.

36. Chao Y, Li CP, Chao TY, Su WC, Hsieh RK, Wu MF, et al. An open, multi-centre, phase II clinical trial to evaluate the efficacy and safety of paclitaxel, UFT, and leucovorin in patients with advanced gastric cancer. Br J Cancer 2006;95:159-63.

37. Okines AF, Norman AR, McCloud P, Kang YK, Cunningham D. Meta-analysis of the REAL-2 and ML17032 trials: evaluating capecitabine-based combination chemotherapy and infused 5-fluorouracil-based combination chemotherapy for the treatment of advanced oesophago-gastric cancer. Ann Oncol 2009;20:1529-34.

38. Pazdur R, Lassere Y, Rhodes V, Ajani JA, Sugarman SM, Patt YZ, et al. Phase II trial of uracil and tegafur plus oral leucovorin: an effective oral regimen in the treatment of metastatic colorectal carcinoma. J Clin Oncol 1994;12:2296-300.

39. Eng C. Toxic effects and their management: daily clinical challenges in the treatment of colorectal cancer. Nat Rev Clin Oncol 2009;6:207-18. 